\title{
Improving the performance of solar panels by the use of phase-change materials
}

\author{
Pascal Biwole ${ }^{1, *}$, Pierre Eclache ${ }^{2}$, Frederic Kuznik ${ }^{2}$ \\ ${ }^{1}$ University of Nice Sophia-Antipolis, Nice, France \\ ${ }^{2}$ University of Lyon, Villeurbanne, France \\ *Corresponding author. Tel: +33 492965029, Fax: +33 492965071, E-mail:phbiwole@unice.fr
}

\begin{abstract}
High operating temperatures induce a loss of efficiency in solar photovoltaic and thermal panels. This paper investigates the use of phase-change materials (PCM) to maintain the temperature of the panels close to the ambient. The main focus of the study is the CFD modeling of heat and mass transfers in a system composed of an impure phase change material situated in the back of a solar panel (SP). A variation of the enthalpy method allows simulating the material thermo-physical change of properties. The buoyancy term in Navier-Stokes' momentum conservation equation is modified through an additional term which forces the velocity field to be non-existent when the PCM is solid. For validation purposes, isotherms and velocity fields are calculated and compared to those from an experimental set-up. Results show that adding a PCM on the back of a solar panel can maintain the panel's operating temperature under $40^{\circ} \mathrm{C}$ for around two hours under a constant solar radiation of $1000 \mathrm{~W} / \mathrm{m}^{2}$.
\end{abstract}

Keywords: Solar Panel, Operating Temperature, Phase Change Material

\section{Nomenclature}

\begin{tabular}{|c|c|c|c|}
\hline$C p$ & specific heat.... & $P$ & pressure. \\
\hline & gravitational constant ............................. $\mathrm{m} \mathrm{s}^{-2}$ & $T_{m}$ &..$K$ \\
\hline & PCM container height ................................ $m$ & $u$ &.$m \cdot s^{-1}$ \\
\hline & thermal conductivity.. & $\Delta T$ & of melt \\
\hline$u_{F}$ & Latent heat of fusion .. & $\beta$ & ansion.... \\
\hline & PCM cont & $\rho$ & density. \\
\hline & mass .... & & 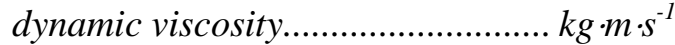 \\
\hline
\end{tabular}

\section{Introduction}

The efficiency of solar panels depends on three factors: the intensity of the solar radiation flux, the quality of the semi conductor in use, and the operating temperature of the semi conductor cell. The variations of solar radiation cannot be controlled. Therefore the ongoing research focuses either on new material like copper, indium diselenium, cadmium tellurium and chalcopyrites, or on maintaining low operating temperatures. For PV panels, high operating temperatures create a drop in the conversion rate of about $0.5 \%$ per Celsius degree over the nominal cell operating temperature of $25^{\circ} \mathrm{C}$ [1], as defined by the industry standard STC (Standard Test Conditions). In summer, panel's temperature typically ranges from 40 to $70^{\circ} \mathrm{C}$ which makes a 7.5 to $22.5 \%$ drop in the conversion rate. In the same way, the efficiency of solar thermal panels decreases mainly because of radiation losses when their operating temperature is above the ambient.

To lower the operating temperature, one can either improve the free cooling on the back of the panel thanks to natural or forced convection, or try to absorb the excess heat by modifying the panel's architecture. The latter solution includes the use of PCMs situated on the back of solar panels. PCMs are materials that undergo reversible transition of phase depending on their temperature. They absorb or reject heat in the process. Only a few studies have been specifically devoted to passive cooling of solar panels by SP/PCM architectures. The hypothesis driving the research is simple: when the panels' temperature rises, the excess heat 
must be absorbed until the PCM has completely melted. When the panel's temperature decreases, the solidification of the PCM should provide additional heat for the operating liquid in solar thermal panels, provide heat to the building or act as an insulation material. The SP/PCM solution is expected to be very useful for roof or facade integrated panels where space for ventilation is limited.

Huang et al. [2] studied the melting of PCMs in an aluminum container submitted to a solar radiation of 750 to $1000 \mathrm{~W} / \mathrm{m}^{2}$. They used a finite volume model to resolve both the heat transfers diffusion and the Navier-Stokes equations. They later included cooling fins in the tank to improve the PCM bulk thermal conductivity [3] [4]. They found that the temperature rise in the system could be reduced by more than $30^{\circ} \mathrm{C}$ for 130 minutes. Cellura et al. [5] resolved the same architecture using a finite element PDE solver. However, they considered the PCM as pure, meaning that the PCM melting temperature is unique and does not change while the PCM is still melting. This property is not valid for most commercial PCMs which are generally mixtures of several different materials. By resolving only the heat transfers diffusion equation, they showed that a PCM with a melting temperature between $28^{\circ} \mathrm{C}$ and $32^{\circ} \mathrm{C}$ can improve the energy conversion efficiency by around $20 \%$ in summer time. Jay et al. [6] experimentally studied a layout where PCM were contained in a honeycomb grid to improve conduction in the container. They showed that after 6 hours and 30min of experiment under an artificial insulation of $800 \mathrm{~W} / \mathrm{m}^{2}$ on real PV panels, the temperature of a PV/PCM system was still lower than that of a single panel, with a mean temperature difference of $24^{\circ} \mathrm{C}$. They also found that the panel's temperature drop using a PCM with a melt temperature at $27^{\circ} \mathrm{C}$ was higher than using a PCM with a melt temperature at $45^{\circ} \mathrm{C}$.

In this study, we consider the same geometry as [2]. The transient conduction and convection heat transfers as well as the Navier-Stokes equations are simultaneously resolved in the PCM domain using a finite element model on a fixed grid. The buoyancy term in Navier-Stokes' momentum conservation equation is modified through an additional term to force the velocity field to be zero when the PCM is solid. This scheme is validated using an experimental set-up. The model is then used for a parametric study of the SP/PCM architecture performances.

\section{Methodology}

\subsection{Numerical case description}

The geometry of the model is presented in Fig. 1. The thermo-physical properties of the simulated materials are presented in Table 1.

Table 1. Thermo-physical properties of RT25 and aluminum.

\begin{tabular}{cccc}
\hline & $\mathrm{Cp}$ & $\mathrm{k}$ & $\rho$ \\
\hline Solid RT25 & 1800 & 0.19 & 785 \\
Liquid RT25 & 2400 & 0.18 & 749 \\
Aluminum & 903 & 211 & 2675 \\
Constant properties of RT25: \\
$\mathrm{L}_{\mathrm{F}}: 232000 \mathrm{~J} \mathrm{~kg}^{-1}$ \\
$\mathrm{~T}_{\mathrm{m}}: 26.6^{\circ} \mathrm{C}, \Delta \mathrm{T}=1^{\circ} \mathrm{C}$ \\
$\beta: 1 \mathrm{e}-3 \mathrm{~K}^{-1}$ \\
$\mu: 1.7976 \mathrm{e}-3 \mathrm{~m}^{2} . \mathrm{s}^{-1}$ \\
\hline
\end{tabular}

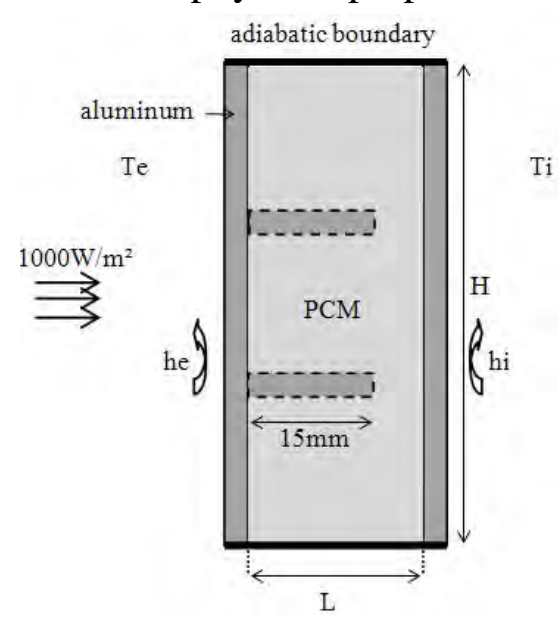

Fig. 1. Geometry of the numerical model. 


\subsection{Mathematical model}

\subsubsection{Modeling heat transfers}

Over the front plate surface, we considered conduction, convection and radiation heat transfers as shown in Eq. (1). Long wave radiation with the sky was neglected in the model.

$\rho C_{p} \frac{\partial T}{\partial t}=-k \frac{\partial T}{\partial x}+h_{e}\left(T_{e}-T\right)+\alpha E(t)$

where $\alpha$ is the aluminum thermal absorptivity and $\mathrm{E}$ the solar radiation intensity. The heat transfers diffusion equation applies over the PCM, the air layer and the aluminum domains:

$\rho C_{p} \frac{\partial T}{\partial t}+\nabla \cdot(-k \nabla T)+\rho C_{p} \vec{u} . \nabla T=0$

The velocity field $\boldsymbol{u}$ in Eq. (2) is given by Navier-Stokes equations for incompressible fluids. To model the changes in PCM RT25 thermo-physical properties occuring during the phase transition, we define function $\mathrm{B}_{0}$ as the liquid fraction in the PCM domain. Let be $\mathrm{T}_{\mathrm{m}}$ the mean melt temperature and $\Delta \mathrm{T}$ the half range of melt temperatures:

$$
B_{0}(T)=\left\{\begin{array}{ccc}
0 & , & T<\left(T_{m}-\Delta T\right) \\
\left(T-T_{m}+\Delta T\right) /(2 \Delta T) & , & \left(T_{m}-\Delta T\right) \leq T<\left(T_{m}+\Delta T\right) \\
1 & , & T>\left(T_{m}+\Delta T\right)
\end{array}\right.
$$

Eqs. (3) show that $B_{0}$ is zero when the PCM is in solid and 1 when it is in liquid phase. $B_{0}$ linearly grows from zero to 1 between the two states. To ensure second order continuous differentiability of the liquid fraction over the temperature domain and to help numerical convergence, $B_{0}$ is approximated by a second order differentiable function $B_{1} \cdot B_{1}(T)$ is the sixth-degree polynomial whose coefficients are calculated using the following conditions:

$$
\left\{\begin{array}{lllll}
B_{1}\left(T_{m}-\Delta T\right)=0 & ; & B_{1}^{\prime}\left(T_{m}-\Delta T\right)=0 & ; & B^{\prime \prime}{ }_{1}\left(T_{m}-\Delta T\right)=0 \\
B_{1}\left(T_{m}+\Delta T\right)=1 & ; & B_{1}^{\prime}\left(T_{m}+\Delta T\right)=0 & ; & B^{\prime \prime}{ }_{1}\left(T_{m}+\Delta T\right)=0
\end{array}\right.
$$

where $\mathrm{B}_{1}$ ' and $\mathrm{B}_{1}$ " are $\mathrm{B}_{1}(\mathrm{~T})$ first and second derivatives. $\mathrm{B}_{1}$ is used to model the changes in the PCM thermo-physical properties as follows:

$$
\begin{aligned}
& \rho(T)=\rho_{\text {solid }}+\left(\rho_{\text {liquid }}-\rho_{\text {solid }}\right) \cdot B_{1}(T) \\
& k(T)=k_{\text {solid }}+\left(k_{\text {liquid }}-k_{\text {solid }}\right) \cdot B_{1}(T)
\end{aligned}
$$

The modeling of the specific heat includes an additional term representing the latent heat of fusion absorbed during the melting process:

$$
\begin{aligned}
& C_{p}(T)=C_{p_{\text {solid }}}+\left(C_{p_{\text {liquid }}}-C_{p_{\text {solid }}}\right) \cdot B_{1}(T)+L_{F} \cdot D(T) \\
& \text { Where } \quad D(T)=e^{\frac{-T\left(T-T_{m}\right)^{2}}{\Delta T^{2}}} / \sqrt{\pi \cdot \Delta T^{2}}
\end{aligned}
$$


Function D is a smoothed Delta Dirac function which is zero everywhere except in interval $\left[T_{m}-\Delta T, T_{m}+\Delta T\right]$. It is centered on $T_{m}$ and its integral is 1 . Its main role is to distribute the latent heat equally around the mean melting point.

\subsubsection{Modeling mass transfers}

We assumed that the PCM in the liquid phase is a Newtonian fluid. The mass, momentum and energy conservation equations were resolved simultaneously with the heat transfers diffusion equation. However, to model the phase transition, the momentum conservation equation was modified as follows:

$\rho \frac{\partial \vec{u}}{\partial t}+\rho(\vec{u} . \nabla) \vec{u}-\mu \cdot \nabla^{2} \vec{u}=-\nabla P+\vec{F}_{b}+\vec{F}_{a}$

where $\mathbf{F}_{\mathbf{b}}$ is buoyancy force given by the Boussinesq approximation:

$\vec{F}_{b}=-\rho_{\text {liquid }}\left(1-\beta\left(T-T_{m}\right)\right) \vec{g}$

And $\vec{F}_{a}=-A(T) \cdot \vec{u}$

with $A(T)=\frac{C\left(1-B_{1}(T)\right)^{2}}{\left(B_{1}(T)^{3}+q\right)}$

The expression of $\mathrm{A}$ is inspired from the Carman-Kosensky relation in a porous medium where the value of $\mathrm{C}$ depends on the morphology of the medium. If we assume that the flow is laminar:

$\nabla(P)=\frac{-C(1-B(T))^{2}}{B_{1}(T)^{3}} \cdot \vec{u}$

In this study, $\mathrm{C}$ is given the constant value $10^{5}$. This value is chosen arbitrarily high. Constant $q$ is chosen very low in order to make Eq. (12) valid even when $B_{1}(T)$ is zero. The value of $q$ was fixed at $10^{-3}$. When the temperature of the MCP is higher than $T_{m}+\Delta T$, the PCM is completely liquid. Therefore, $\mathrm{B}_{1}$ is 1 and consequently, $\mathrm{A}$ and $\mathbf{F}_{\mathbf{a}}$ are zero. In this case, the usual momentum conservation equation applies. During the transition state, $0<\mathrm{B}_{1}(\mathrm{~T})<1$. $\mathrm{A}(\mathrm{T})$ increases along with the melting process until the added force $\mathbf{F}_{\mathbf{a}}$ becomes greater than the convection and diffusion terms in Eq. (9). The momentum conservation equation becomes similar to the Darcy law for fluid flow in porous medium:

$\vec{u}=-\frac{K}{\mu} \nabla(P)$

where the permeability $K$ is a function of $B_{1}(T)$. When $B_{1}(T)$ diminishes, the velocity field also diminishes until it reaches zero when the PCM becomes completely solid. At that point, the MCP temperature is lower than $T_{m}-\Delta T$. Therefore, $B_{1}$ is 0 . Eq. (12) shows that the value of $\mathrm{A}(\mathrm{T})$ becomes very high. Consequently, all the terms in the momentum conservation equation are dominated by the added force. The only solution of the Navier-Stokes equations is $\mathbf{u}=\mathbf{0}$ which corresponds to a solid medium. 


\subsubsection{Numerical method}

A 2D finite element model was used. To satisfy the Brezzi-Babuska condition [7] three degrees of freedom were used on each element to approximate the pressure field and an additional degree of freedom was used by adding a node at the center of mass of each triangular element to approximate the velocity field. The maximum mesh size was $4.10 \mathrm{e}-4 \mathrm{~m}$. The final mesh had 135 240 elements and 465857 degrees of freedom. No significant change of the results was observed when using a finer mesh. A Galerkin least-squares stabilization method was employed.

\subsection{Elements of validation}

\subsubsection{Experimental set-up}

The experimental set-up consisted of a $167 \mathrm{~mm} \mathrm{x}$ 167mm x30mm-large Plexiglass container without cooling fins and filled with a PCM. An air layer was left at the top of the tank to prevent it from breaking due to the PCM thermal expansion. A

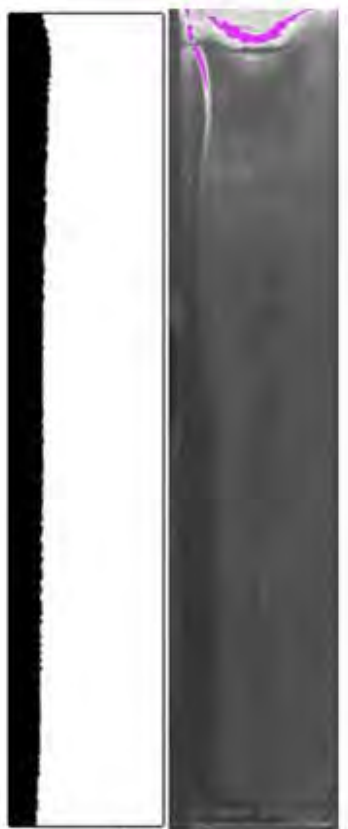

$20 \mathrm{~min}$

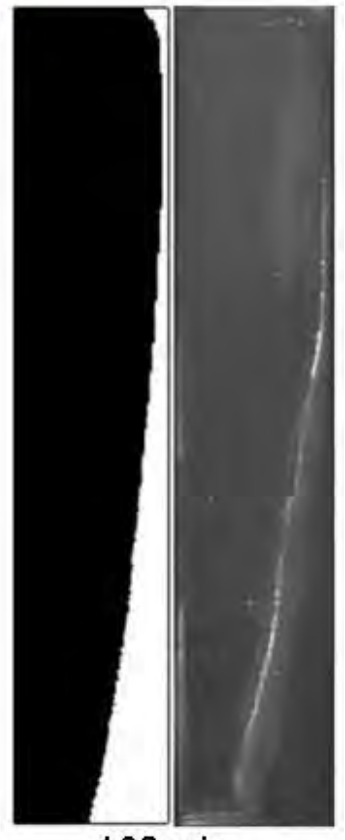

$160 \mathrm{~min}$
Fig. 2. Comparison of the simulated (left) and the experimental(right) liquid-solid moving boundary location fixed temperature of $\mathrm{Te}=20^{\circ} \mathrm{C}$ and $\mathrm{Ti}=40^{\circ} \mathrm{C}$ was imposed on each side of the tank thanks to heating plates. The transient two-dimensional velocity field in the tank was measured thanks to a PIV apparatus including a Nd-Yag laser.

The experimental validation consisted of: firstly, a transient comparison of the moving liquidsolid boundary location (Fig. 2); secondly, a comparison of the simulated and measured velocity fields in the completely melted PCM (Fig. 3). Some discrepancies were noted between the simulated and the measured velocity fields. This may be due to the very low velocities in the tank which are of the order of $10 \mathrm{e}-4 \mathrm{~m} / \mathrm{s}$. However, the transient locations of the simulated moving boundary closely matched the experimental one.

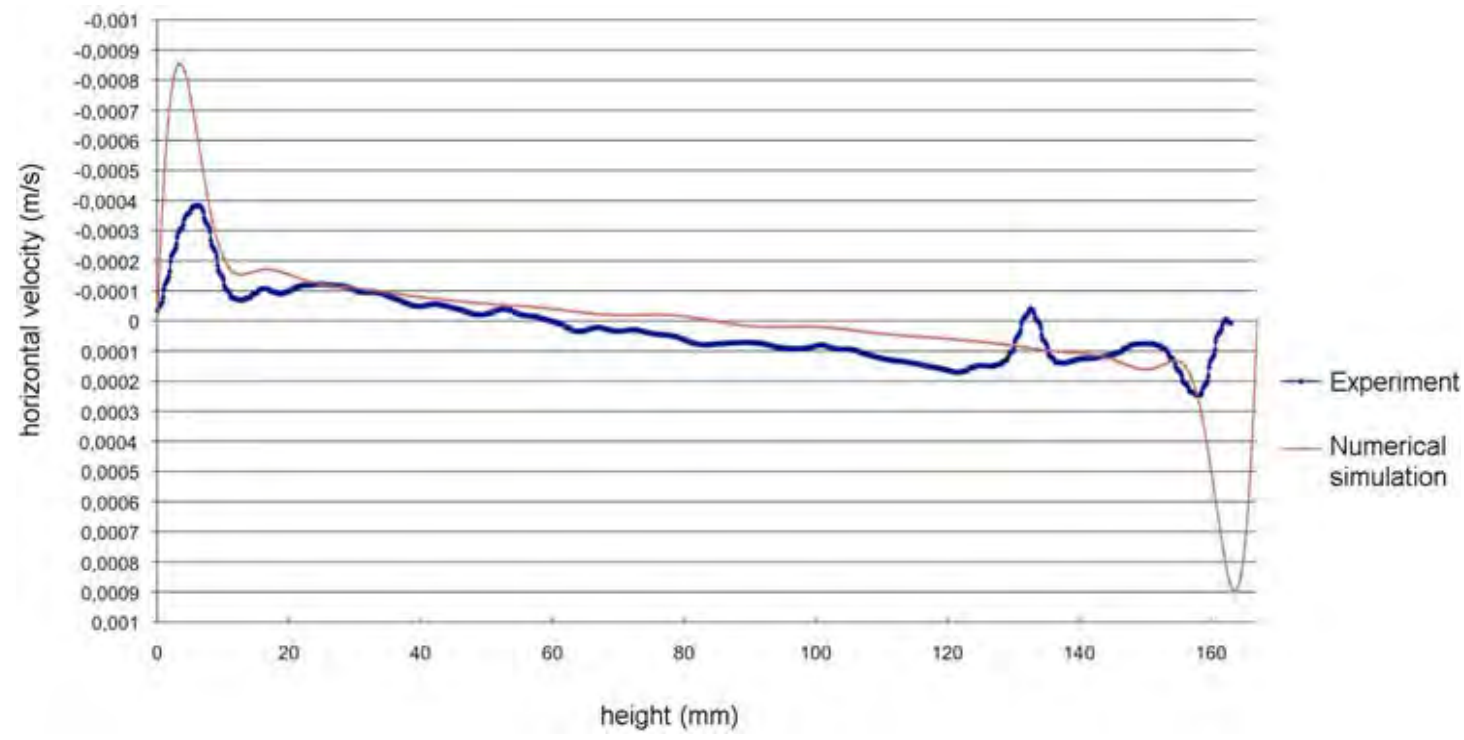

Fig. 3. Comparison of the PIV-measured and simulated velocity in the vertical mid cross-section of the PCM domain. 


\section{Results}

All the simulations were conducted using he $=10 \mathrm{~W} / \mathrm{m}^{2} \cdot \mathrm{K}, \mathrm{hi}=5 \mathrm{~W} / \mathrm{m}^{2} \cdot \mathrm{K}$ and $\mathrm{E}=1000 \mathrm{~W} / \mathrm{m}^{2}$.

\subsubsection{Temperature and velocity fields}

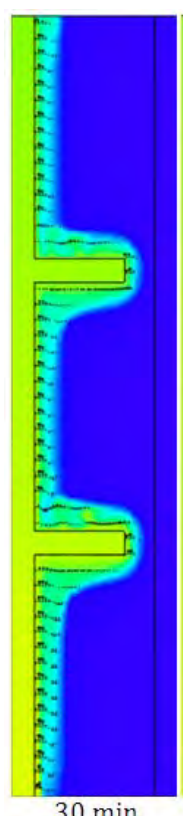

$30 \mathrm{~min}$

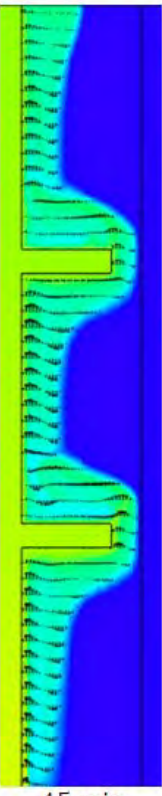

$45 \mathrm{~min}$

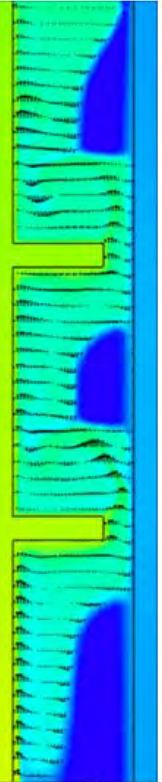

$65 \mathrm{~min}$

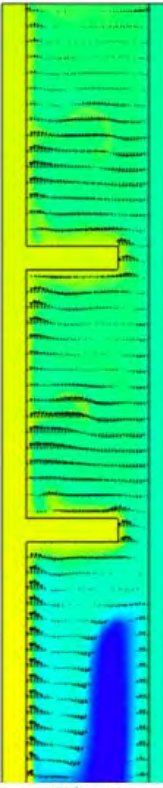

$75 \mathrm{~min}$

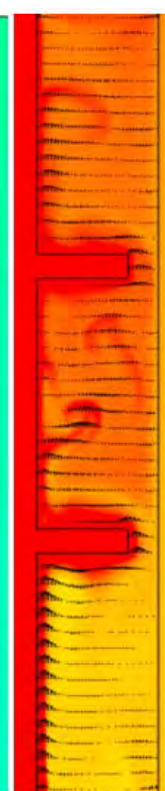

$89 \mathrm{~min}$

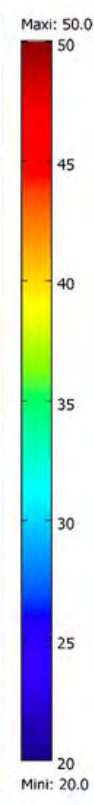

Mini: 20.0

Fig. 4. Simulated transient isotherms and velocity fields in the SP/PCM system. $H=132 \mathrm{~mm}, L=20 \mathrm{~mm}$.

\subsubsection{Parametric study}

Table 2. List of simulated cases.

\begin{tabular}{ccccc}
\hline & (a) & (b) & (c) & (d) \\
\hline $\mathbf{L}(\mathbf{m})$ & 0 & 20 & 20 & 20 \\
$\mathbf{H}(\mathbf{m})$ & 132 & 132 & 40 & 132 \\
Cooling fins & no & yes & no & no \\
\hline
\end{tabular}

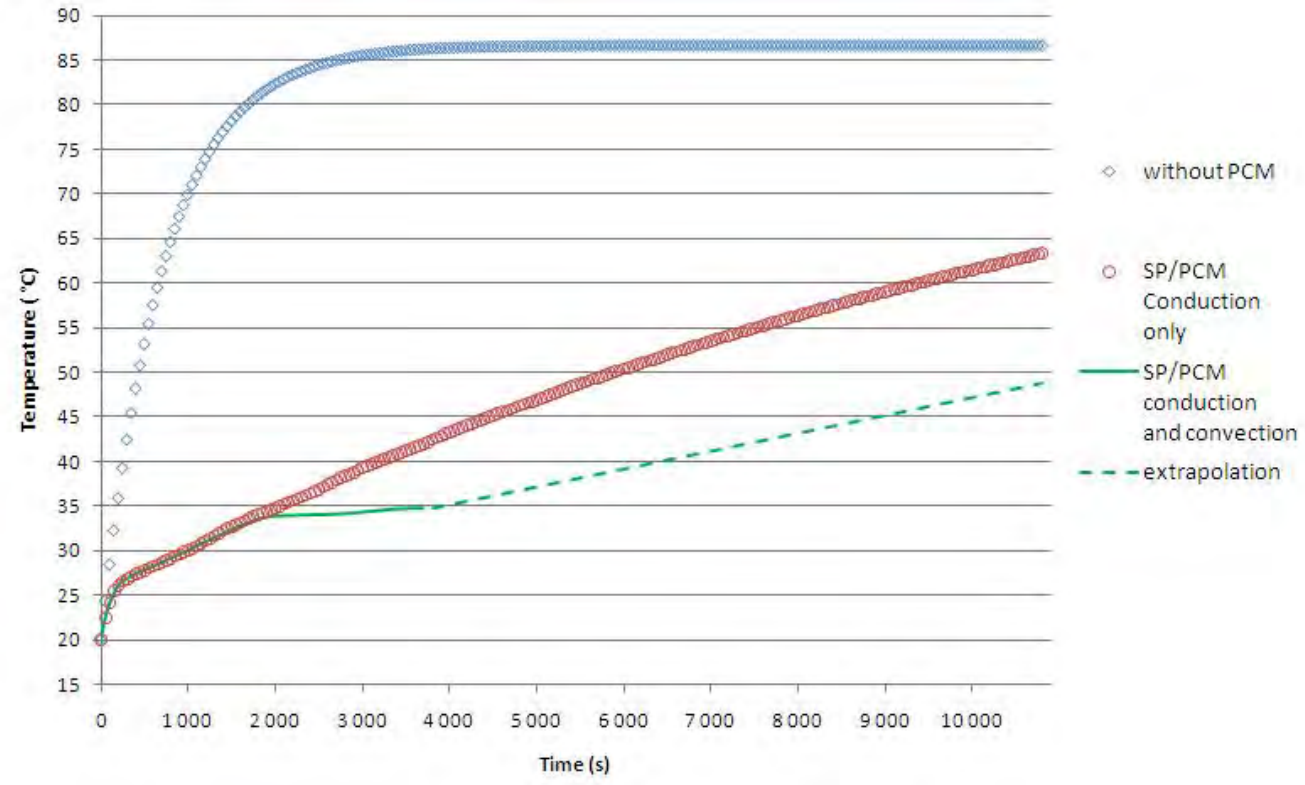

Fig. 5. Impact of convection heat transfers in the PCM on the panel's operating temperature. $H=0.132 \mathrm{~m}$ and $L=0.049 \mathrm{~m}$. 


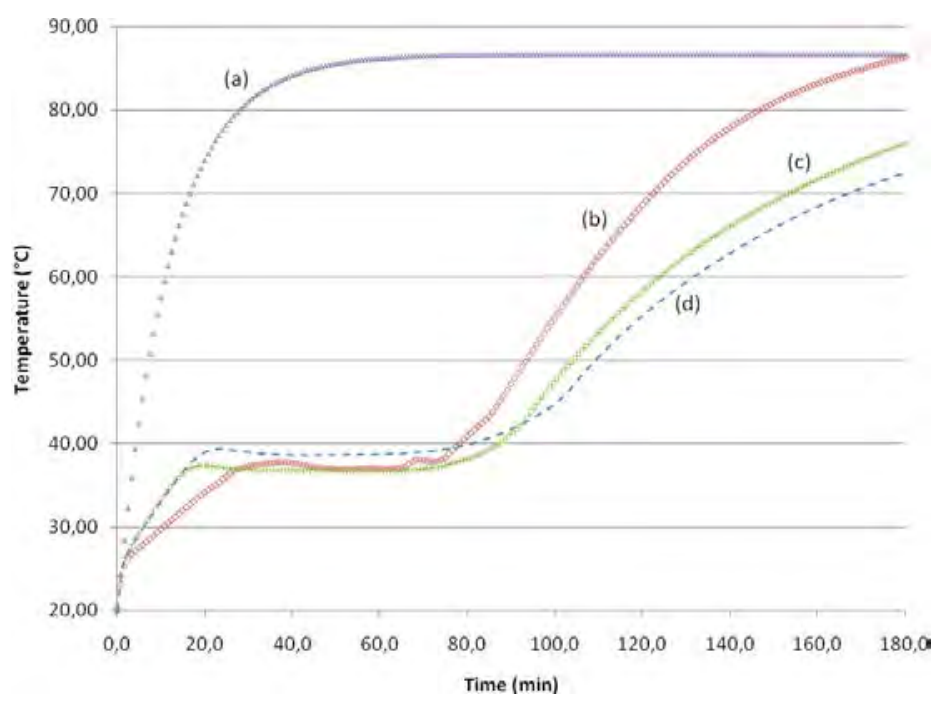

Fig. 6. Impact of the SP/PCM size on the panel's operating temperature.

\section{Discussion and conclusion}

The first limitation of this work rises from the representation of the solar panel by an aluminum plate. This simplification does not take into account the bulk specific capacity of real panels. The second limitation comes from the fact that the impact of sky temperature was not included in the numerical model, due to experimental validation difficulties. Despite those limitations, the following observations remain relevant:

The velocity field in Fig. 4 shows a circulation of the liquid PCM through the three parts of the container. Convection of the liquid PCM is observed upward close to the heated panel, and downward close to the liquid-solid boundary. The Rayleigh number in the liquid PCM was between $10^{5}$ at melt start and $10^{6}$ at melt end. This result is coherent with our initial assumption of a laminar flow and confirms the observation of [4]. Like [4], we also numerically observe a suspended solid PCM mass when the melting is nearly over.

Over the range of simulated sizes for the SP/PCM system, the temperature of the front plate always remains lower than $50^{\circ} \mathrm{C}$ after $89 \mathrm{~min}$ under a constant radiation of $1000 \mathrm{~W} / \mathrm{m}^{2}$. The better performance is obtained with a $13.2 \times 4.9 \mathrm{~cm}$ large PCM container. In this case, the panel's temperature is $34.9^{\circ} \mathrm{C}$ after 1 hour. The same temperature is reached after $5 \mathrm{~min}$ without PCM (Fig. 5). These observations are in good agreement with [3] and [5]. Three inflexions points can be observed on the transient operating temperature profile. The first one happens at the PCM melt temperature. After a steep increase, the panel temperature rises much more slowly from that point because of the start of the melting process.

Between the first and the second inflexion point, the PCM acts like an insulation material for the panel and heat transfers are dominated by conduction (Fig. 5). The second inflexion point indicates the start of the convection heat transfers which balances conduction heat transfers in the PCM. Fig. 5 shows that the simulated panel temperature may be overestimated by $20 \%$ after 3600s when conduction only is considered in the numerical model. On Figs. 5 and 6, the operating temperature remains more or less constant until the PCM has completely melted. The last inflexion point marks the end of the melting process. Heat transfers in the container are dominated by convection. Afterwards, the temperature rises more slowly than before the start of the melt because of the higher specific heat capacity of the liquid PCM. 
The parametric study also shows that the operating temperature drops proportionally to the increase of the $\mathrm{PCM}$ width: after $3600 \mathrm{~s}, \mathrm{~T}=34.9^{\circ} \mathrm{C}$ when $\mathrm{L}=0.049 \mathrm{~m}$ whereas $\mathrm{T}=37^{\circ} \mathrm{C}$ when $\mathrm{L}=0.02 \mathrm{~m}$. Comparing curves (c) and (d) shows that the same trend is observed when the panel height is increased but only when the PCM has completely melted. In brief, it is better to increase the PCM width than its height to lower the panel's temperature. Adding cooling fins in the PCM tank provides a faster attenuation of the operating temperature because the PCM bulk conductivity is increased. But this layout accelerates the phase transition too. When the PCM has completely melted, the operating temperature rises faster than for all other SP/PCM architectures (Fig. 6). This fact moderates the idea that adding cooling fins makes SP/PCM systems more efficient [4].

To conclude, adding PCM on the back of solar panels is an efficient way of improving panels' performance. Their operating temperature can be substantially decreased using that technology. Future work should include experimental validations of this first numerical model using real solar panels under real climate.

\section{References}

[1] K. Emery, J. Burdick, Y. Caiyem, D. Dunlavy, H. Field, B. Kroposki, T. Moriatry, Temperature dependence of photovoltaic cells, modules and systems, Proceedings of the 25th IEEE PV Specialists Conference, Washington DC, USA, May 13-19, 1996, pp. $1275-1278$.

[2] M.J. Huang, P.C. Eames, B. Norton, Thermal regulation of building integrated photovoltaics using phase change materials, International Journal of Heat and Mass Transfers 47, 2004, pp. 275-2733.

[3] M.J. Huang, P.C. Eames, B. Norton, Phase change materials for limiting temperature rise in building integrated photovoltaics, Solar Energy 80 (9), 2006, pp. 1121-1130.

[4] M.J. Huang, P.C. Eames, B. Norton, Comparison of a small-scale 3D PCM thermal control model with a validated 2D PCM thermal control model, Solar Energy Materials and Solar Cells 90 (13), 2006, pp. 1961-1972.

[5] M. Cellura, G. Ciulla, V. Lo Brano, A. Marvuglia, A. Orioli, Photovoltaic panel coupled with a phase changing material heat storage system in hot climates. In: Proceedings of the 25th Conference on Passive and Low Energy Architecture, Dublin, Ireland, October 2224, 2008.

[6] A. Jay, S. Clerc, B. Boillot, A. Bontemps, F. Jay, Utilisation de matériaux à changement de phase pour réduire la température de panneaux PV intégrés au bâti, Proceedings of the International Building Performance Simulation Association conference, Moret sur Loing, France, November 9-10, 2010.

[7] D. N. Arnold, F. Brezzi, M. Fortin, A stable element for the Stokes equations, Calcolo (21), 1984, pp. 337-344. 